\title{
Résilience communautaire et participation paysanne à l'évaluation des risques à Madagascar : importance de l'approche participative
}

Community resilience and peasant participation in risk assessment in

Madagascar : importance of the participatory approach

Resiliencia comunitaria y participación campesina en la evaluación de riesgos en Madagascar : importancia del enfoque participativo

Hermann K. LEMENA, Jaurès C RALITERA, Julien Salava et Mahefasoa Randrianalijaona

\section{(2) OpenEdition \\ 1 Journals}

\section{Édition électronique}

URL : http://journals.openedition.org/ctd/4021

DOI : $10.4000 /$ ctd. 4021

ISSN : 2491-1437

Éditeur

Chaire Unesco Pratiques émergentes en technologies et communication pour le développement

Édition imprimée

ISBN : 2497-1437

\section{Référence électronique}

Hermann K. LEMENA, Jaurès C RALITERA, Julien Salava et Mahefasoa Randrianalijaona, « Résilience communautaire et participation paysanne à l'évaluation des risques à Madagascar : importance de l'approche participative ", Communication, technologies et développement [En ligne], 9 | 2021, mis en ligne le 26 mars 2021, consulté le 01 avril 2021. URL : http://journals.openedition.org/ctd/4021 ; DOI : https://doi.org/10.4000/ctd.4021

Ce document a été généré automatiquement le 1 avril 2021. 


\section{Résilience communautaire et participation paysanne à l'évaluation des risques à Madagascar : importance de l'approche participative}

Community resilience and peasant participation in risk assessment in

Madagascar : importance of the participatory approach

Resiliencia comunitaria y participación campesina en la evaluación de riesgos en Madagascar : importancia del enfoque participativo

Hermann K. LEMENA, Jaurès C RALITERA, Julien Salava et Mahefasoa Randrianalijaona

1 Chaque année à Madagascar, les communautés sont victimes des catastrophes engendrées par les aléas naturels majeurs tels que le cyclone, l'inondation, la sécheresse et le glissement de terrain, suite aux dégâts qu'ils causent sur leur passage. Les conséquences varient d'une région à l'autre et dépendent non seulement de l'intensité de l'aléa, mais aussi de la vulnérabilité des actifs, en particulier, la population et sa capacité à y résister. Les empreintes laissés par ces différents aléas sur la population malagasy rendent fragile l'économie locale déjà précaire. Souvent, les communautés rurales sont les plus affectées en raison de leurs activités économiques fortement exposées aux chocs.

2 Une évaluation approfondie des risques de catastrophes contribuerait à la mise en place d'une solution durable et au renforcement de la résilience de la population.

Cette évaluation consiste à s'assurer que les éléments, les activités, les situations et les processus entre autres qui peuvent causer un préjudice à des personnes ou à des biens sont maîtrisés. Des études participatives communautaires permettraient de promouvoir des pratiques durables et d'accélérer l'échange d'opinions entre différents 
acteurs. C'est une approche qui préconise une implication active du public aux processus décisionnels. Il s'agit donc d'un processus qui invite au pluralisme, à la diversité et à la différence d'opinions, avec pour ambition d'examiner les questions à partir d'un maximum d'angles pour trouver la meilleure solution commune (Slocum et al. 2006).

4 Dans les pays en développement comme Madagascar, la pratique politique favorise généralement la mise en œuvre de l'approche descendante ou « top down ». Elle est au service des décideurs et oblige les échelles de compétence à suivre les lignes directives venant d'en haut. Toutefois, l'approche descendante, sans concertation avec les bénéficiaires finaux, est sans effet significatif sur le niveau de vie des populations (Lebovics, 2007). Par contre, l'approche participative a été conçue afin d'inciter la participation active de tout un chacun et d'atténuer la portée de l'approche habituelle «top down"; d'où la question: "Comment la méthode ERBC contribue-t-elle au renforcement de la résilience communautaire? » Le présent article consiste à mettre en application l'Évaluation des Risques à Base Communautaire (ERBC) dans deux régions qui ont des caractéristiques complètement différentes. L'objectif de cet article est de capitaliser les apports bénéfiques de la participation communautaire à la gouvernance des risques et des catastrophes à partir des études menées dans la région SAVA et Atsimo Andrefana de Madagascar.

\section{Concept de la Résilience}

5 Le domaine de la résilience n'est pas un terrain en friche et le concept le sous-tendant n'est donc pas nouveau. En effet la résilience figure parmi les concepts fondamentaux de la Gestion des Risques et des Catastrophes (GRC) et du développement suite à une série de catastrophes dans le monde. Le défi majeur auquel nous devrions faire face est la détermination des moyens pour rendre le système résilient afin d'assurer leur durabilité (Paquet, 1999). Dans le domaine de l'écologie, Holling (1973) a introduit le concept de «systèmes résilients » selon lequel, la résilience est définie comme «la capacité de l'environnement à absorber les perturbations et à se réorganiser pendant le changement tout en gardant essentiellement la même fonction, même structure, même identité et même rétroactions ». Ensuite, elle transverse dans d'autres domaines comme les sciences sociale, humaine, économique, etc. Paquet (1999) considère la résilience comme «la capacité intrinsèque des entreprises, des organisations et des communautés à retrouver un état d'équilibre ».

6 Le Bureau des Nations Unies pour la réduction des risques de catastrophe (UNISDR) adopte également une définition similaire à celles déjà citées, considérant ainsi la résilience comme : "la capacité d'un système, d'une communauté ou d'une société exposée aux risques de résister, d'absorber, d'accueillir et de corriger les effets d'un danger, en temps opportun et de manière efficace, notamment par la préservation et la restauration de ses structures essentielles et de ses fonctions de base » (UNISDR, 2009, p. 27).

7 En somme, la résilience d'un système peut être définie comme sa capacité à intégrer des changements suite à un choc, tout en conservant ses propres caractères. 


\section{Pourquoi adopter une approche participative ?}

8 Depuis la moitié des années 80 , les approches participatives sont devenues un outil important pour un processus de prise de décision plus efficace et productif. Elles sont appliquées dans plusieurs secteurs. L'engagement des populations et des acteurs, souvent négligés, dans le processus permet de générer l'information et de révéler des préférences sociétales.

9 Les membres d'une communauté locale ont généralement une meilleure compréhension des préoccupations et problèmes locaux que le gouvernement et les autres acteurs de développement ignorent, malgré leurs manques de connaissances techniques concernant les politiques à adopter. Cette approche leur offre la possibilité de discuter des forces et des faiblesses des politiques à adopter et à mettre en valeur les problèmes à résoudre (Whitmore, Guijt, et al. 2006, cité in Jacob et Ouvrard, 2009). Cependant, des compétences extérieures peuvent être demandées pour aider la communauté à comprendre les questions d'ordre technique afin que les membres puissent y participer efficacement. Par conséquent, les décisions ou les politiques adoptées dans le cadre de l'approche participative seront plus aisément acceptées par la société, car la prise de décision est basée sur des modes consensuels et les décisions prises sont cohérentes à la situation réelle et à la culture de la société (Zuckerman, et al. 2003). Les résultats obtenus reposent sur des faits solides et des informations concrètes (Jacob et Ouvrard, 2009).

Sur le processus décisionnel, les approches participatives peuvent entraîner des lenteurs (Baron et Monier, 2003), mais l'efficacité et la durabilité des résultats sont largement reconnues, car elles permettent de construire des relations solides dans la confiance. L'approche participative crée une atmosphère de confiance, d'autocritique et d'entraide réciproque qui permet aux participants de partager plus aisément leurs difficultés et leurs problèmes (Whitmore, Guijt, et al. 2006, cité in Jacob et Ouvrard, 2009). Elle améliore considérablement la qualité des décisions en rendant le processus plus efficace et plus constructif.

11 Nous avons choisi la méthode participative pour évaluer la vulnérabilité des ménages ruraux par rapport aux aléas naturels, car elle correspond aux besoins des études sociales ou culturelles à mener, facilite le choix des politiques à adopter et permet facilement de clarifier les problèmes avant de formuler des solutions adéquates. Ainsi, pour évaluer la vulnérabilité des ménages ruraux face aux cyclones et aux sécheresses, nous avons recouru à la méthode participative dénommée «Évaluation des Risques à Base Communautaire (ERBC)».

\section{Approche méthodologique}

12 Tout d'abord, l'évaluation des risques consiste à analyser les risques menaçant la population et ses biens ainsi que ses infrastructures afin de mettre en place une politique de sécurisation et de sureté envers la communauté.

Une méthode participative interactive à l'identification et à l'évaluation des risques par la communauté a été adoptée afin de réduire sa vulnérabilité dans un processus de prise de décision et d'adoption des mesures spécifiques locales. Le principal objectif de cette méthode est d'aider les communautés à comprendre les risques qu'ils encourent 
et d'évaluer le degré de leur vulnérabilité afin d'éviter sa transformation en catastrophes. En se servant de la méthode d'évaluation à base communautaire des risques, les participants arrivent à une même compréhension du profil du risque.

Nous avons commencé notre investigation par l'établissement d'une cartographie communautaire en vue de localiser le terrain. Une formation axée sur la connaissance des outils d'évaluation du risque comme l'identification des aléas, l'arbre à problème et à solution, le diagramme de Venn, et le calendrier saisonnier a par la suite été dispensée auprès des communautés locales en vue de leur permettre de participer aux échanges.

Le focus group est la base de la méthodologie dans l'utilisation des outils de l'ERBC. En tant qu'acteur principal du focus, il nous est revenu les rôles d'organisateur, d'animateur, d'observateur et de facilitateur. Dans ce cas, la participation effective de tout un chacun est nécessaire, sans distinction ni de niveau d'études ou de connaissances, ni d'âge ou de sexe. Toutefois, la sélection repose sur la qualité hétérogène de la composition afin de panacher les points de vue.

Ainsi, nous avons choisi des catégories socio-professionnelles des participants telles que maires, médecins, agriculteurs, instituteurs, etc. La démarche entreprise était participative afin que toutes les entités concernées par le développement de la zone aient été impliquées pour assurer le plus large diagnostic possible de la situation réelle de la zone d'étude.

Le focus group nous a permis de recueillir les informations relatives à l'implication des participants. Dans la région SAVA, 8 focus groupes ont été réalisés durant le mois de janvier et février 2018. Le nombre de participants par Fokontany varie entre 15 à 20 chefs de ménage. Pour la région Atsimo Andrefana, 4 focus groupes de 15 personnes ont été effectués, courant le mois de septembre 2017.

\section{Présentation et choix des zones d'études}

\section{Région SAVA}

18 La région SAVA se situe sur la partie Nord-Est de Madagascar. La population pratique généralement les cultures vivrières pour subvenir à sa consommation et les cultures de rentes constituent la principale source de revenu des ménages. La région SAVA est fortement exposée au risque de cyclone. Entre 1960 et 2016, 51 cyclones l'ont touchée et y ont laissé des lourdes conséquences. Ce sont en majorité des cyclones tropicaux intenses. En mars 2017, un cyclone de catégorie 4 a frappé la région (Randrianalijaona, 2019). Une stratégie de réduction des risques basée sur la perception et la compréhension locale est nécessaire.

19 En effet, l'étude a été effectuée dans 4 communes rurales du district de Sambava et d'Antalaha. Ces districts sont les plus exposés aux cyclones dans la région SAVA. Le choix de ces quatre communes repose principalement sur leur situation géographique et l'importance du dommage causé par le passage de ce dernier cyclone. 


\section{Région Atsimo Andrefana}

20 Ankililoaka est une commune rurale abritant 62192 Habitants $^{1}$ dans le district de Toliara II, région Sud-Ouest de Madagascar. Elle est fortement exposée aux divers problèmes environnementaux et aux sécheresses. Cette commune est la limite sud de la forêt sèche des Mikea qui abrite nombreuses espèces aussi bien floristiques que faunistiques endémiques. Ces espèces sont pourtant exposées à des grandes menaces de disparition, résultant d'un côté des pressions anthropiques et d'un autre côté de la vulnérabilité du milieu naturel. L'accès à la commune étant relativement facile grâce à la route nationale RN9. Les principales activités des ménages sont les cultures vivrières et la culture du Coton.

\section{Résultats}

21 L'ERBC a permis aux communautés et aux chercheurs d'identifier ensemble les effets des aléas naturels et des vecteurs qui les renforcent sur les conditions de vie des ménages. Ces aléas affectent essentiellement les habitats, la santé, les infrastructures et l'agriculture.

22 Dans le secteur agricole, les calendriers culturaux sont perturbés à cause de changement et bouleversement provoqués par les aléas climatiques. Dans la région SAVA, le calendrier de culture coïncide avec la saison cyclonique. À Madagascar, les cultures restent tributaires du climat et des méthodes traditionnelles. Par conséquent, la sècheresse et le cyclone diminuent fortement les productions et les rendements agricoles d'une année à l'autre où l'autonomie alimentaire de la population est à craindre. Tandis que dans la région du Sud-Ouest, suite à la sécheresse récurrente, la communauté tout entière a remarqué l'apparition des nouveaux insectes, qui détruisent des variétés de plantes cultivées. En 2016, environ 10 ha de terrain de culture irriguée, en phase de maturité, a été détruit par ces insectes nuisibles à Ankililoaka. Après la sécheresse, le rendement et la production agricole ont fortement diminué.

En matière de gouvernance des risques, l'évaluation permet également de comprendre les rôles de tous les organismes présents au sein de la communauté. Les participants peuvent dresser la liste des acteurs présents dans leurs communautés même si certains d'entre eux ont été souvent négligés. Ils sont généralement composés des comités de GRC, des ONG, des organismes gouvernementaux, des institutions internationales et nationales, des associations, des collectivités territoriales, des médias et des paysans. Malgré l'existence de comité local de GRC dans certaines localités, la majorité de la population ignore encore l'existence de ces comités.

24 Pour le cas des habitations, le type de matériaux utilisés pour la construction constitue le facteur de vulnérabilité de la population au cyclone et à la sècheresse. Dans la région SAVA, en milieu rural, la plupart des maisons sont en matériaux artisanaux et ne résistent pas aux cyclones, même de catégorie 1 . Les zones d'habitations qui se trouvent sur les flancs Est des collines sont les plus exposées au cyclone. À Ankililoaka, la plupart des habitations ne suivent pas les normes de construction et fabriquées à base de matériaux locaux qui absorbent des vagues de chaleur, défavorables à la vie quotidienne de la population. 
Concernant les infrastructures routières, dans la Région SAVA, elles restent encore insuffisantes, en mauvais état et difficilement accessible durant les périodes de pluie. Le passage de cyclone renforce le problème d'accessibilité. Il entraine une hausse importante des prix des produits de première nécessité, surtout dans les villages très enclavés. L'infrastructure d'eau potable est quasi inexistante dans les milieux ruraux. Les sources d'approvisionnement en eau sont généralement non aménagées. En cas de passage de cyclone, elles sont devenues inutilisables, car les défécations s'y sont versées. Et dans la Région Sud-Ouest, durant les saisons sèches, plusieurs ménages n'ont plus accès à l'eau potable.

La faible perception du risque et le manque de préparation constituent un facteur majeur de destruction des habitations et de décès ainsi que de la difficulté d'adaptation de la population après le choc.

Quelques solutions sont proposées à l'issue de l'initiative des participants pour faire face aux problèmes rencontrés lors de son évaluation. Les efforts basés sur l'amélioration de la production visant à accroître les revenus agricoles pour mieux résister aux catastrophes en vue d'atteindre des niveaux croissants de résilience constituent leurs principales propositions.

\section{Discussion}

8 Depuis des décennies, le cyclone représente un évènement majeur qui cause souvent des catastrophes importantes dans la région SAVA contre la sècheresse dans le sud. Ces aléas provoquent souvent des dégâts et pertes inestimables dus au fort degré de vulnérabilité de la population. À Madagascar, la GRC repose encore sur les mesures réactives pour sauver des vies humaines et aider les victimes dans leur relèvement. De plus, les autorités locales sont souvent internées dans le système top down qui ne laisse que peu de place pour intégrer les initiatives locales. De ce fait, l'approche top down renforce les conditions de vulnérabilité du fait qu'elle ne tient pas compte les connaissances locales et que les victimes deviennent dépendantes des aides extérieures. Cette situation a fait appel à l'approche participative qui reconnait la nécessité d'impliquer les membres de la communauté dans la GRC dans leurs communautés.

D'après le profil historique dessiné par la communauté, des cyclones à forte intensité frappent la région SAVA presque tous les 10 ans environ et la population d'Ankililoaka souffre de la sècheresse presque toute l'année depuis les années 60. L'approche participative interactive, notamment l'ERBC, apparait appropriée pour comprendre la vulnérabilité des populations rurales face aux cyclones et aux sècheresses, car elle permet d'appréhender les opportunités et tous les points qui pourraient fragiliser les populations rurales. Cette étude a confirmé le résultat d'étude de Hutter et al. (2013) et de Mahmood et. Mahbub (2018). Les riches informations recueillies au niveau de la communauté a permis de comprendre profondément leurs pratiques et leur perception des risques qu'elle encourt. Mertens (1999) a souligné que l'inclusion de personnes marginalisées permet de collecter des données plus adaptées. L'analyse dynamise la discussion entre les participants à travers les problèmes actuel et futur et rassemble la perception des risques par les communautés, qui va forcément accentuer leurs perceptions du risque et leur résilience. 
30 Malgré une généralisation de la manifestation de la vulnérabilité, notre recherche a démontré que les communautés sont habilitées à exposer explicitement leurs difficultés et à y apporter les solutions appropriées, moyennant une approche participative, engageant les décideurs dans l'analyse des risques auxquels ces dernières sont exposées. La méthode implique la communauté dans les processus décisionnels de politiques publiques, qui se sont accrus de manière considérable actuellement (Beuret et Cadoret, 2010). L'ERBC, comme toutes les études participatives (Torres, Stone, et al. 2000 ; Butterfoss, Francisco, et Capwell, 2001 ; Abma, 2005 ; Whitmore, Guijt, et al. 2006 ; Plante et al. 2011; Somda, Sawadogo et al. 2014), est flexible, ce qui facilite sa réalisation et permet de mettre en place un processus faisable et acceptable par tous. D'ailleurs, à l'échelle internationale, cette approche est recommandée dans le domaine de GRC.

31 La connaissance approfondie des difficultés des ménages ruraux et la reconnaissance des solutions qu'ils proposent jouent un rôle important à la prévention et la préparation aux risques et aussi à l'organisation communautaire en cas de catastrophe. Les savoirs locaux vont pousser les connaissances scientifiques à l'élaboration d'une réflexion commune pour comprendre les problèmes de la communauté et ainsi pour leur résolution conjointe par de consensus de décisions prises. Les communautés auront envisagé plus des solutions à leurs problèmes grâce au transfert de connaissances scientifiques. Et, cela contribue significativement au renforcement de la résilience de la communauté et de manière durable en raison de son engagement sur le long terme dans les politiques mises en place (Charlotte et al. 2012).

\section{Conclusion}

L'évaluation à base communautaire des risques effectuée dans les milieux ruraux aide les paysans à identifier les aléas auxquels les communautés sont exposées, car ces derniers pourraient provoquer des dégâts sur les cultures et les infrastructures et perturbe la vie quotidienne de la communauté. L'efficacité d'une telle approche réside au niveau de son applicabilité qui s'avère facile à comprendre pour les ménages à partir du moment où ils sont conscients des risques qui les menacent. Elle favorise également la pratique de la démocratie participative à travers l'implication de la population dans toutes les démarches de gouvernance des risques. Toutefois, les participants principalement les membres du Fokontany ont besoin de renforcer systématiquement leur capacité sur l'utilisation de cette approche afin qu'ils puissent rendre facile l'activité d'évaluation.

Les outils ont permis aux participants de répertorier les acteurs qui interviennent dans la communauté pour surmonter l'évènement, d'identifier le calendrier du risque, de déterminer les problèmes liés à un aléa, ses effets et ses conséquences probables qui sont généralement ou habituellement minorés ou ignorés par la communauté et d'initier des actions pour renforcer la prévention, suivant leurs propres compréhensions et perceptions des risques et cela afin de réduire leurs vulnérabilités.

Les solutions préconisées sont axées principalement sur la préparation aux évènements et l'amélioration des revenus. Pendant les discussions, certains participants partagent les bonnes pratiques aux stratégies d'adaptation à adopter. L'approche participative permet d'augmenter la connaissance des risques et de réduire la vulnérabilité. Elle contribue fortement au renforcement de la résilience de la communauté rurale. 
Malgré les avantages de cette approche, elle a des limites. Les principales difficultés reposent sur le plan organisationnel surtout la logistique et la pratique, notamment sur le temps, les ressources financières ou humaines, l'organisation et les compétences des acteurs.

\section{BIBLIOGRAPHIE}

Abma T. A. (2005). Responsive Evaluation : Its Meaning and Special Contribution to Health Promotion. Evaluation and Program Planning 28(3), pp. 279-289.

Alam E. and Collins A. E. (2010). Cyclone disaster vulnerability and response experiences in coastal Bangladesh. Overseas Development Institute, Disasters, 34(4), pp. 931-954.

Beuret J.-E. et Cadoret A. (2010). Gérer ensemble les territoires : Vers une démocratie coopérative. Paris : Éditions Charles Léopold Mayer.

Bherer L. (2011). Les relations ambigües entre participation et politiques publiques. De Boeck Supérieur, Participations $\mathrm{n}^{\circ}$ 1, pp. 105-133.

Butterfoss F. D., Francisco V. and Capwell E. (2001). Stakeholder Participation in Évaluation. Health Promotion Practice 2(2), pp. 114-119.

Charlotte D. C., Steve P. et Liette V. (2012). Le suivi de la résilience de communautés côtières comme moyen d'évaluation des effets de la recherche-action participative. Université de Québec à Rimouski, Département Sociétés, Territoires et Développement et Brock University, Department of Biological Sciences. Vertigo, volume 18, numéro 2.

Dagdeviren H., Donoghue M. and Promberger M. (2016). Resilience, hardship and social conditions. Journal of Social Policy 45, 1, pp. 1-20.

Goulden M.C., Adger W. N., Allison E. H., and Conway D. (2013). Limits to Resilience from Livelihood Diversification and Social Capital. Lake Social-Ecological Systems. Annals of the Association of American Geographers 103, pp. 906-924

Holling C. S. (1973). Resilience and stability of ecological systems. Annual Review of Ecological Systems, pp. 1-23.

Hutter G., Kuhlicke C., Glade T. and Felgentreff C. (2013). Natural hazards and resilience : exploring institutional and organizational dimensions of social resilience. Nat Hazards 67 : pp. 1-6. Jacob S. et Ouvrard L. (2009). Comprendre et entre prendre une évaluation participative. Guide de synthèse. Edition de l'Université de Laval, Québec, 32p

Khan A. A. (1974). Perception of cyclone hazard and community response. Oriental Geographer, pp. 1-7.

Klein R. J., Nicholls R. J. and Thomall F. (2003). Resilience to natural hazards : how useful is this concept? Environ Hazards 5, pp. 35-45.

Lebovics M. (2007). Analyse des apports et des contraintes du développement participatif. Afrique contemporaine/3 ( $\left.\mathrm{n}^{\circ} 223-224\right)$, pp. 403-432. 
Mahmood R. and Mahbub A. Q. M. (2018). Building Vulnerable Islander Resilience to Natural Hazard : A Participatory Approach. Handbook of Climate Change Resilience.

Mertens D. M. (1999). Inclusive Evaluation : Implications of Transformative Theory for Evaluation. American Journal of Evaluation 20(1), pp. 1-14.

Plante S., Chouinard O. et Martin G. (2011). Gouvernance participative par l'engagement citoyen à l'heure des changements climatiques : études de cas à Les Goulet, Point-du-Chêne et Bayshore Drive (Nouveau-Brunswick). Territoire en mouvement, Revue de géographie et aménagement, 11, pp. 33-49.

Randrianalijaona (2019). Measuring the economic impact of cyclones in Madagascar, https:// theconversation.com/measuring-the-economic-impact-of-cyclones-in-madagascar-90946

SAMBISOA M. J., LEMENA K. H. et BIN ISSOUF H. (2018). Plan Communal de Développement Ankililoaka. BE DELMARA, 261p

Rowe G. et Frewer L. J. (2000). Public participation methods : A framework for evaluation. Science, Technology, \& Human Values 25(1), pp. 3-29.

Slocun N., Elliot J., Heersterbeek S. and Lukensmeyer C. (2006). Méthodes participatives : un guide pour l'utilisateur. Bruxelles : Fondation Roi Baudouin.

Torres R. T., Stone S. P. et al., Butkus D. L., Hook B. B., Casey J., Arens S. A. (2000). Dialogue and Reflection in a Collaborative Evaluation : Stakeholder and EvaluatorVoices. New Directions for Evaluation (85), pp. 27-38.

UNISDR, 2009, Terminologie pour la Prévention des risques de catastrophe, Nations Unies, Génèves, Suisse, 39 p, https://www.unisdr.org/files/7817_UNISDRTerminologyFrench.pdf

Walker J., and Cooper M. (2011). Genealogies of Resilience : From Systems Ecology to the Political Economy of Crisis Adaptation. Security Dialogue, 42(2), pp. 143 - 160.

Ward N. et Percy F. (2015). Adaptation à base communautaire : une approche d'autonomisation pour un développement résilient au climat et la réduction des risques. Copyright : CARE International, CARE Climate Change edition, 9 p. http://www.careclimatechange.org/adaptation-initiatives

Whitmore, E., Guijt, I. et, Mertens M. D., Imm P., Chinman M., Wandersman A. (2006). Embedding Improvements, Lived Experience, and Social Justice in Evaluation Practice. Handbook of Evaluation : Policies, Programs and Practices. Edited by Ian F. Shaw, Jennifer C. Greene, Melvin M. Mark. London : Thousand Oaks, Californie, Sage Publications.

\section{NOTES}

1. Commune rurale d'Ankililoaka, octobre 2017.

\section{RÉSUMÉS}

La collaboration entre les chercheurs et la communauté de base en matière de gestion des risques naturels est une relation déjà établie depuis plusieurs années. Pour une meilleure évaluation des 
risques au niveau communautaire, plusieurs méthodes d'analyse comme les critères standards d'évaluation et la recherche-action en sciences sociales ont souvent été utilisées. Mais ces derniers temps, une méthode d'évaluation des risques à base communautaire est développée pour améliorer la fiabilité des résultats d'analyse par diverses raisons. Cette nouvelle méthode est pratiquée par plusieurs pays et différents organismes ou entité travaillant dans le domaine des risques de catastrophe. Le présent article soulève l'importance de l'utilisation, par l'animateur, de quelques outils de l'évaluation des risques à base communautaire jugés pertinents pour une analyse à partir des contextes locaux.

The collaboration between researchers and the grassroots community in the management of natural hazards is a relationship that has already been established for several years. To be able to assess risks at community level, several analysis methods such as standard assessment criteria and action research in social science have often been used. However, in recent times, a community-based risk assessment method has been developed to improve the reliability of the analysis results for various reasons. This new method is used by several countries and by different organizations or entities working in the field of disaster risks. This article highlights the importance of the facilitator's use of some community-based risk assessment tools deemed relevant in order to be able to perform the analysis based on local contexts and the general objective of the research.

La colaboración entre los investigadores y la comunidad de base en la gestión de los riesgos naturales es una relación que se ha establecido desde hace muchos años. Para evaluar los riesgos a nivel comunitario, se han utilizado con frecuencia varios métodos de análisis, como los criterios de evacuación estándar y la investigación de acción cientificos sociales. Sin embargo, en los últimos tiempos se está desarrollando un método del riesgo basado en la comunidad para mejorar la fiabilidad de los resulatados analíticos por diversas razones.este nuevo método es practicado por varios países y diferentes organizaciones o entidades que trabajan en el campo del riesgo de desastres. Este artículo destaca el uso, por l'animador, algunas herramientas de evaluación des riesgos basadaos en la comunidad que se consideren pertinentes para los contextos locales y el objectivo general de la investigación.

\section{INDEX}

Palabras claves : evaluación, riesgo, resiliencia, vulnerabilidad, comunidad, Madagascar

Mots-clés : Évaluation, risques, résilience, vulnérabilité, communauté, Madagascar

Keywords : Assessment, risk, resilience, vulnerability, community, Madagascar

\section{AUTEURS}

\section{HERMANN K. LEMENA}

Doctorant en Agronomie, École doctorale Gestion des Ressources naturelles et Développements, Université d'Antananarivo (UA). Centre d'Études et de Recherches Economiques pour le Développement, UA

\section{JAURÈS C RALITERA}

Doctorant en Sciences économiques, École Doctorale Sciences humaines et sociales, UA. Centre d'Études et de Recherches Economiques pour le Développement, UA. 


\section{JULIEN SALAVA}

Maître de Conférences à la Mention Economie, UA Centre d'Études et de Recherches Economiques pour le Développement, UA

MAHEFASOA RANDRIANALIJAONA

Centre d'Études et de Recherches Economiques pour le Développement, UA 